\title{
STEADY PLANE MHD FLOWS THROUGH POROUS MEDIA IN THE MAGNETOGRAPH PLANE
}

\section{C.S. Bagewadi* and S. Bhagya**}

\section{Abstract}

We obtain solutions for steady plane MHD flow through porous media when velocity and magnetic vectors are constantly and variably inclined and the magnitude of the magnetic vector is constant on each individual stream line in the magnetograph plane. If is shown that the path of magnetic and velocity vectors are circles congruency to each other. Also flow analysis is carried out by writing the expression of Legendre transformation in polar co-ordinates. It is shown that solutions obtained agree with the graphs.

Key words : Stream line, Magnetic line, Legendre transformation, Magnetograph plane.

\section{Introduction}

Flow of a viscous liquid in a porous medium is of great and increasing importance in the study of percolation through soils in hydrology, petroleum industry and in agricultural engineering. The flows in porous media generally

*- Dean, Foculty of Science \& Technology, Chairman-Department of Mathematics \& Computer Science, Kuvempu University, Jnana Sahyadri, -577 451, Shimoga, Karnataka, India.

** Department of Mathematics, P.E.S. College, Bangalore-560 050, India. 
involve extensively low Reynolds numbers and such flows are called Darcy.flows. Such flows are valid under several limitations. It is shown that it can be possibly valid only in a certain seepage velocity domain outside which more general equations may be used to describe the flow. This happens because initial effects become important. Also the flow through porous boundaries is of great importance both in technological as well as bio-physical fields, examples of which are soil mechanics, transpiration cooling, food preservation, cosmetic industry, blood flow and artificial dialysis. In recent years the problem of fluid flow past porous media or in channels with mass transfer, heat transfer have gained more importance because of varied application. For example, I.V.fluid containers made of PVC are commonly used these days. Water from inside permeates out thus increasing the concentration of drug inside and sometimes becoming hazardous to life. Therefore, the study relating to suction or injection is very important: The early researchers considered the blood to be a Newtonian fluid but being a suspension of cells it behaves as a nonNewtonian fluid at low shear rates in small arteries.

O.P. Chandna and his co-workers $[1,3,10,14,15]$ have published a series of papers on plane incompressible MHD flow of a viscous fluid. They have obtained solutions for these flows by transforming the basic equations from cartesion plane to velocity plane by using Legendre transformation. K.K. Singh \& D.P. Singh [8] studied the solutions in variably inclined MHD plane flows in porous media. C.S. Bagewadi and Siddabasappa $[6] ;[7]_{;}[9],[11],[12]$ extended the work of above authors and published a series of papers on MGD flow, EMFD flow and Rotating MHD flow. They have obtained solutions for these flows by transforming the basic equations from cartesion plane to velocity and magnetograph planes. These methods infact help to study the flows in a more general way by the use of Jacobian matrix. Yamamofa [4],[13] examined the flow past porous bodies by applying the generalised law using the generalised momentum equations. Ram and Mishra [2] have studied the unsteady MHD flow of fluid through a porous medium in a circular pipe under action of a consant pressure gradient.

In the present paper, we study MHD flow of a viscous incompressible fluid of infinite electrical conductivity through porous media when (i) angle between $\mathrm{V}$. and $\mathrm{H}$ is constant (ii) the magnitude of magnetic vector is constant on each individual magnetic line in the magnetograph plane. In the 2 nd section basic equations are written and are decomposed in the cartesion plane. The 3rd section deals with some preliminaries about magnetograph plane. In the 4 th section equations written in cartesion plane for constantly inclined flows are recast into magnetograph plane and flow analysis is carried out. In the 5th section the equations for MHD flow when the magnitude of the magnetic lines are constant are recast in the magnetograph plane and flow analysis is carried. The results obtained in our 
paper are entirely different and infact extensions from the results obtained by the above authors. Hence our results are superior to the results obtained by the above authors. Also various graphs are plotted and it is concluded that these graphs agree with the theoritical results obtained.

\section{BASIC EQUATIONS}

The steady MHD flow of a viscous incompressible fluid of infinite electrical conductivity through porous media is governed by [8].

$\operatorname{div} \mathrm{V}=0$

$\rho\left[(v \cdot g r a d) M=-\operatorname{grad} P+\eta \nabla^{2} V+\mu J X H-(\mu / K) V\right.$

$\operatorname{Curl}(\mathrm{VXH})=0$

$\operatorname{div} \mathrm{H}=0$

where $V$ is the Velocity vector, $H$ is the Magnetic field vector, $P$ is the Pressure function, $r$ is the Constant density, $\eta$ is the Constant co-efficient of Viscosity, $\mu$ is the Constant magnetic permeability, $K$ is the Permeability of the medium and $J$ is the Current densify.

We consider the flow to be the two dimensional so that $\mathrm{V}$ and $\mathrm{H}$ lie in a plane defined by the rectangular co-ordinates $(x, y)$ and all the flow variables are functions of $x$ and $y$. Therefore the above system of equations is replaced by the following system

$$
\frac{\partial u}{\partial x}+\frac{\partial v}{\partial y}=0
$$

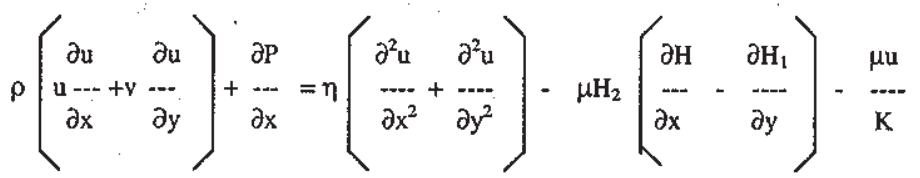

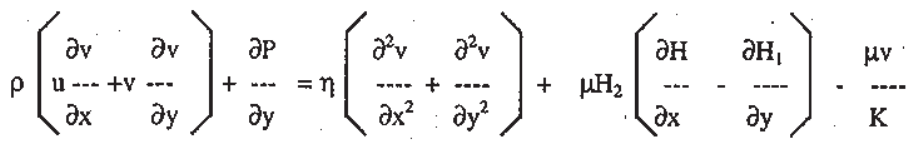

$\mathrm{uH}_{2}-\mathrm{vH}_{1}=\mathrm{k}$

$$
\frac{\partial \mathrm{H}_{1}}{\partial \mathrm{x}}+\frac{\partial \mathrm{H}_{2}}{\partial \mathrm{y}}=0
$$


Now by introducing the functions

$$
\begin{aligned}
\omega . & =\frac{\partial V}{\partial x}-\frac{\partial u}{\partial y} \text { (Vorticity) } \\
j & =\frac{\partial H_{2}}{\partial x} \cdot \frac{\partial H_{3}}{\partial y} \text { (Current density) } \\
h & =P+(1 / 2) \rho q 2 \text { where } q^{2}=u^{2}+v^{2}
\end{aligned}
$$

the above system of equations is replaced (5), (8), (9), (10), (11) and by the following system :

$\eta \frac{\partial \omega}{\partial y} \cdot \rho v \omega+\mu H_{2} j=-\frac{\partial h}{\partial x}-\frac{\mu u}{K}$

(Linear Momentum)

$$
\eta \frac{\partial \omega}{\partial x}-\rho u \omega+\mu H_{j} j=\frac{\partial h}{\partial y}+\frac{\mu v}{K}
$$

of seven non linear partial differential equations in seven unknowns $u, v, H_{1}, H_{2}, \omega$, $i$ and $h$ which are functions of $x, y$. The advantage of this system over to the first system is that it decreased from second order differential equations to the first order differential equations.

\section{Study of Flows in The Magnetograph Plane}

Let the function $H_{1}=H_{1}(x, y) H_{2}=H_{2}(x, y)$ to be such that in the region of flow the Jacobian.

$J(x, y)=\frac{\partial H_{1}}{\partial x} \frac{\partial H_{2}}{\partial y} \frac{\partial H_{1}}{\partial y} \frac{\partial H_{2}}{\partial x} \neq 0 \quad|J|<\infty$

We may consider $x$ and $y$ as functions of $H_{1}$ and $H_{2}$. by means of $x=x\left(H_{1}, H_{2}\right)$ and $y=y\left(H_{1}, H_{2}\right)$ we have the relations,

$$
\frac{\partial H_{1}}{\partial x}=J \frac{\partial Y}{\partial H_{2}}, \frac{\partial H_{1}}{\partial y}=. J \frac{\partial x}{\partial H_{2}}
$$


\begin{tabular}{l}
$\partial \mathrm{H}_{2}$ \\
\hdashline $\mathrm{-}$
\end{tabular}$=-\mathrm{J} \underset{\mathrm{J}}{\frac{\partial \mathrm{y}}{\partial \mathrm{H}_{\mathrm{t}}}} \quad, \quad \frac{\partial \mathrm{H}_{2}}{\partial \mathrm{y}}=\mathrm{J} \frac{\partial \mathrm{x}}{\partial \mathrm{H}_{4}}$

Further more using (16),(17) we have

$f(x, y)=\frac{\partial\left(H_{1}, H_{2}\right)}{\partial(x, y)}=\left[\frac{\partial(x, y)}{\partial\left(H_{1}, H_{2}\right)}\right]^{-1}=J\left(H_{1}, H_{2}\right)$

$\begin{aligned} & \partial f \\ & \partial x\end{aligned}=\frac{\partial(f, y)}{\partial(x, y)}=\frac{\partial(f, y)}{\partial\left(H_{1}, H_{2}\right)}=\bar{J}+\frac{\partial(f, y)}{\partial\left(\mathbf{H}_{1}, H_{2}\right)}$

$\frac{\partial f}{\partial y}=-\frac{\partial(f, x)}{\partial(x, y)}=J \frac{\partial(x, f)}{\partial\left(H_{1}, H_{2}\right)}=\bar{J} \frac{\partial(x, f)}{\partial\left(H_{1}, H_{2}\right)}$

where $f=f(x, y)$ is any continuously differentiable function.

\section{Constantly Inclined Plane Flows}

We now consider constantly inclined plane flows and $f$ denote the constant non zero angle between $v$ and $H$. The vector and scalar products of $V$ and $H$ using the equation (8) yield.

$\mathrm{uH}_{2}-\mathrm{vH}_{1}=\mathrm{qH} \operatorname{Sin} \phi=\mathrm{k}$

$\mathrm{uH}_{1}+\mathrm{vH}_{2}=\mathrm{qH} \operatorname{Cos} \phi=\mathrm{k} \operatorname{Cot} \phi$

where $H=\sqrt{\mathrm{H}_{\mathrm{t}}^{2}+\mathrm{H}_{2}^{2}}$

Solving (2I) and (22) for $\mathrm{U}$ and $\mathrm{v}$ in terms of $\mathrm{H} 1$ and $\mathrm{H} 2$ to get

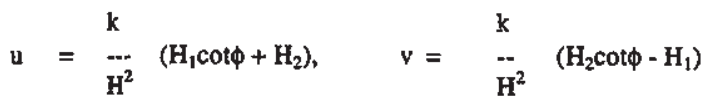

Eliminate functions $u$ and $v$ from the system of equations $(5),(8),(9),(10),(11)$, (13) and (14) by using equation (23) and obtain the following system of six partial differential equations.

$$
\begin{aligned}
& \frac{\partial \mathrm{H}_{1}}{\partial \mathrm{-m}}+\frac{\partial \mathrm{H}_{2}}{\partial \mathrm{x}}=0
\end{aligned}
$$

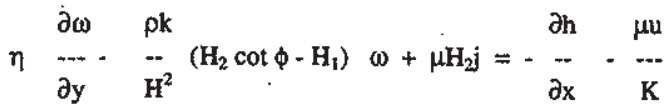


$\eta$\begin{tabular}{ll}
$\partial \omega$ & $\rho \mathrm{k}$ \\
\hline-- & --
\end{tabular}$\underset{\mathrm{H}^{2}}{\left(\mathrm{H}_{1} \cot \phi-\mathrm{H}_{2}\right) \omega+\mu \mathrm{H}_{\mathrm{j}}=} \begin{aligned} & \partial \mathrm{h} \\
& \ddot{\partial y}+\frac{\mu \mathrm{v}}{\mathrm{K}}\end{aligned}$

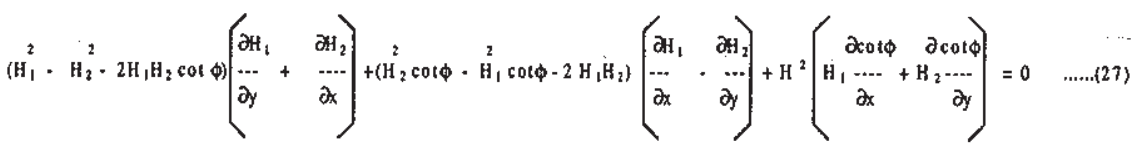

$k \frac{\partial}{\partial x}\left(\begin{array}{c}H_{2} \cot \phi-H_{1} \\ \cdots H^{2}\end{array}\right)-k \frac{\partial}{\partial y}\left(\begin{array}{cc}\left(H_{1} \cot \phi+H_{2}\right. \\ -H^{2}\end{array}\right)=\omega$

$\frac{\partial \mathrm{H}_{2}}{\partial \mathrm{x}}-\frac{\partial \mathrm{H}_{1}}{\partial \mathrm{y}}=\mathbf{j}$

For the six unknown functions $\mathrm{H}_{1}, \mathrm{H}_{2}, \mathrm{j} \omega, \mathrm{h} \phi$ of $(x, y)$ once a solution of this system is determined the velocity vector field is obtained from (23) and then the pressure function is found by using the definition of $h$ in (12). Using the transformation relations (16) to (20) in the equations (24) to (29) we find the following system of equations:

$\frac{\partial x}{\partial \mathrm{H}_{1}}+\frac{\partial \mathrm{y}}{\partial \mathrm{H}_{2}}=0$

$\eta \quad \frac{\partial(\mathrm{x}, \omega)}{\partial\left(\mathrm{H}_{1}, \mathrm{H}_{2}\right)}-\frac{\rho \mathrm{k}}{-\mathrm{H}^{2}}\left(\mathrm{H}_{2} \cot \phi-\mathrm{H}_{1}\right) \omega+\mu \mathrm{H}_{2 j} \mathrm{j}=-\mathrm{J} \quad \begin{gathered}\partial(\mathrm{h}, \mathrm{y}) \\ \ldots \ldots \ldots \\ \partial\left(\mathrm{H}_{1}, \mathrm{H}_{2}\right)\end{gathered}$

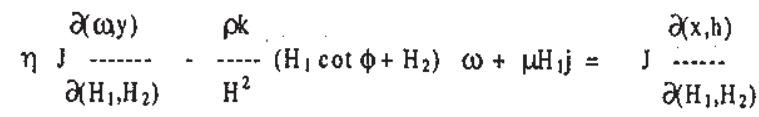

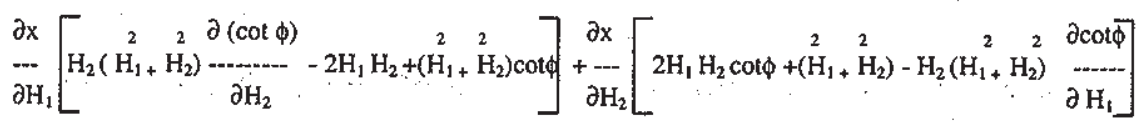

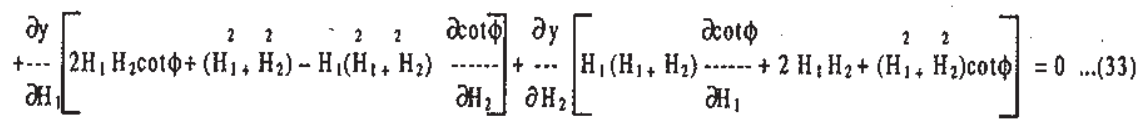

$\mathcal{J}\left[\begin{array}{cc}\partial \mathrm{x} & \partial \mathrm{y} \\ \hdashline \mathrm{H}_{2} & \frac{\partial \mathrm{H}_{1}}{3}\end{array}\right]=\omega$ 


$$
\text { k. } \int\left(\begin{array}{cc}
\left.\partial\left(\mathrm{H}_{2} \cot \phi+\mathrm{H}_{1}\right) / \mathrm{H}_{2}, \mathrm{y}\right) & \left(\partial\left(\mathrm{x}, \mathrm{H}_{1} \cot \phi+\mathrm{H}_{2} / \mathrm{H}_{2}\right)\right. \\
\hdashline \partial\left(\mathrm{H}_{1}, \mathrm{H}_{2}\right) & \partial\left(\mathrm{H}_{1}, \mathrm{H}_{2}\right)
\end{array}\right)=\mathbf{j}
$$

These are six partial differential equations in six unknowns $x\left(\mathrm{H}_{1}, \mathrm{H}_{2}\right)$, y $\left(\mathrm{H}_{1}, \mathrm{H}_{2}\right)$ and four transformed functions w $\left(\left(\mathrm{H}_{1}, \mathrm{H}_{2}\right), \mathrm{h}\left(\left(\mathrm{H}_{1}, \mathrm{H}_{2}\right), \mathrm{i}\left(\mathrm{H}_{1}, \mathrm{H}_{2}\right)\right.\right.$ and $\phi\left(\mathrm{H}_{1}, \mathrm{H}_{2}\right)$. The equation (15) implies the existence of magnetic flux function $Y(x, y)$ such that,

$$
\begin{aligned}
& \partial \Psi \\
& \partial \mathrm{x}
\end{aligned}=-\mathrm{H}_{2}, \quad \frac{\partial \Psi}{-\cdots}=\mathrm{H}_{1}
$$

Likewise, equation (30) implies the existence of a function $\mathrm{L}(\mathrm{H1}, \mathrm{H} 2)$ called the Legendre transform function of the stream function $\Psi(x, y)$ so that,

$$
\frac{\partial \mathrm{L}}{\partial-\mathrm{H}}=-\mathrm{y}, \quad \frac{\partial \mathrm{L}}{\partial \mathrm{H}_{1}}=\mathrm{x}
$$

and the functions $\Psi(x, y)$ and $L(H 1, H 2)$ are related by

$\mathrm{L}\left(\mathrm{H}_{\mathrm{l}}, \mathrm{H}_{2}\right)=\mathrm{H}_{2} \mathrm{x}-\mathrm{H}_{1} \mathrm{y}-\Psi(\mathrm{x}, \mathrm{y})$

Introducing $L\left(\mathrm{H}_{1}, \mathrm{H}_{2}\right)$ into the system (30) to (35) with $\mathrm{J}$ given by (18), it follows that equation $(30)$ is identically satisfied and this system may be replaced by:

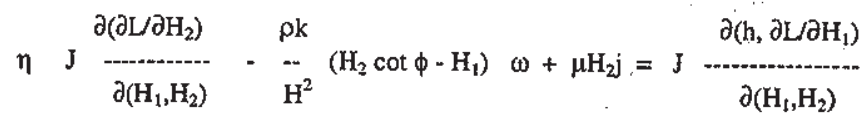

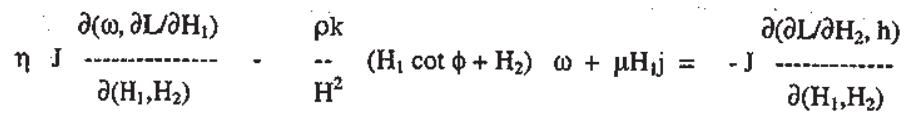

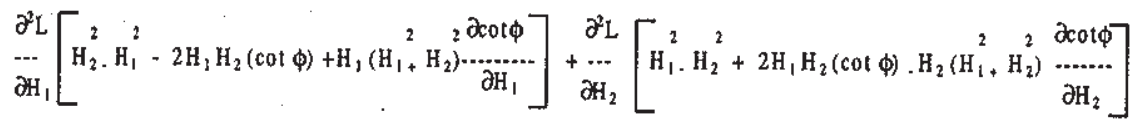

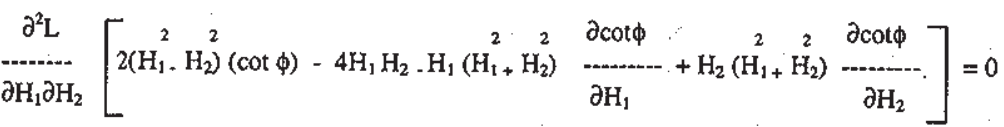

$J\left[\begin{array}{ll}\partial^{2} \mathrm{~L} \\ \hdashline-\partial^{2} \mathrm{~L} \\ \partial \mathrm{H}_{1}\end{array}+\frac{\cdots}{\partial \mathrm{H}_{2}}\right]=w$ 


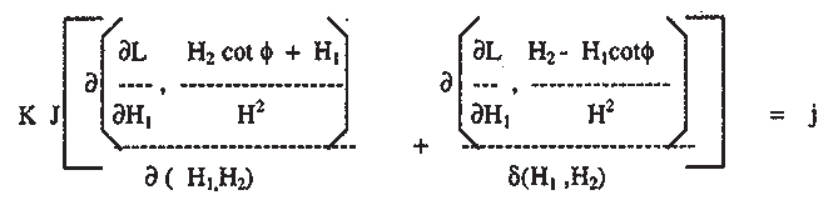

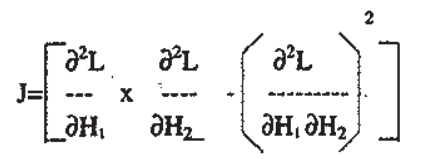

For the six functions $\mathrm{L}(\mathrm{H} 1, \mathrm{H} 2), \mathrm{h}(\mathrm{H} 1, \mathrm{H} 2), \mathrm{i}(\mathrm{H} 1, \mathrm{H} 2), w\left(H 1, \mathrm{H}_{2}\right) J(H 1, H 2)$ and $\mathrm{f}(\mathrm{H} 1, \mathrm{H} 2)$ we define as

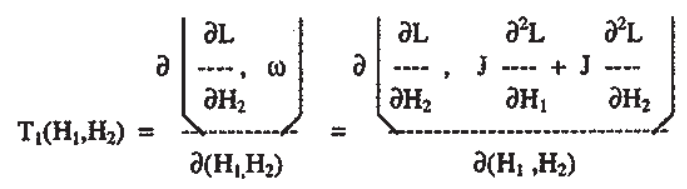

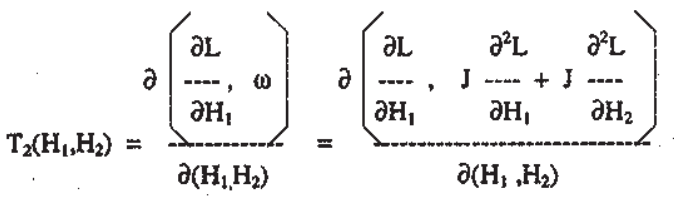

and use the integrability condition

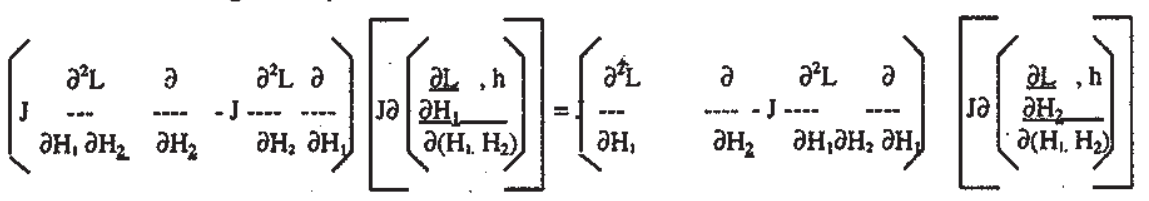

i.e. $\frac{\partial^{2} h}{\partial x \partial y}=\begin{gathered}\partial^{2} h \\ \partial y \partial x\end{gathered}$ to eliminate $h\left(H_{1}, H_{2}\right)$ from equation (39) and (40) to obtain

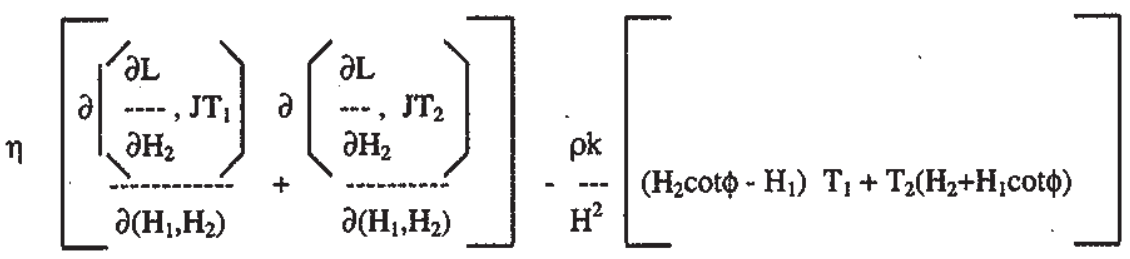




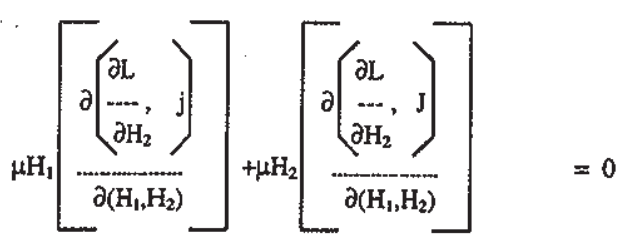

The equations (41)and (47) constitute a system of two non linear partial differential equations in two unknowns $\mathrm{L}(\mathrm{H1}, \mathrm{H} 2), \phi(\mathrm{H1}, \mathrm{H} 2)$ in the Hodograph plane. Once a solution $\mathrm{L}=\mathrm{L}(\mathrm{H} 1, \mathrm{H} 2), \phi=\phi(\mathrm{H} 1, \mathrm{H} 2)$ of equation (41) and (47) is found for which J evaluated from (44) satisfies $0<|\mathrm{J}|<\infty$, the solutions for the magnetic field components $\mathrm{Hl}(\mathrm{x}, \mathrm{y})$ and $\mathrm{H} 2(\mathrm{x}, \mathrm{y})$ are obtained by solving simultaneous equations,

$$
\begin{aligned}
& \partial \mathrm{L} \quad \partial \mathrm{L} \\
& \overline{\partial \mathrm{H}_{1}}=-\mathrm{y}, \quad \overline{\partial-}=\mathrm{x}
\end{aligned}
$$

$$
H=\sqrt{\frac{2}{H_{1}+H_{2}^{2}}} \quad, \theta=\tan ^{-1}\left(H_{2} / H_{1}\right)
$$

or. $H_{1}=H \cos \theta, H_{2}=H \sin \theta$

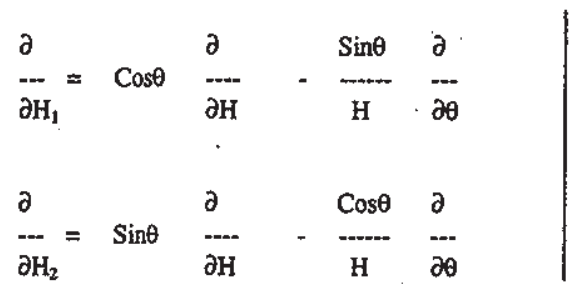

Defining $L^{*}(H, \theta), f^{*}(H, \theta), w^{*}(H, \theta) ; j^{*}(H, \theta), J^{*}(H, \theta)$ to be respectively the Legendre transforms variable angle, vorticity, current density, Jacobean function in $(H, \theta)$ co-ordinates and using. (49) and $(50)$, we have

$$
\frac{\partial(\mathrm{F}, \mathrm{G})}{\partial\left(\mathrm{H}_{1}, \mathrm{H}_{2}\right)}=\frac{\partial\left(\mathrm{F}^{*}, \mathrm{G}^{*}\right)}{\partial(\mathrm{H}, \theta)} \times \frac{\partial(\mathrm{H}, \theta)}{\partial\left(\mathrm{H}_{1}, \mathrm{H}_{2}\right)}=\frac{1}{\mathrm{H}} \frac{\partial\left(\mathrm{F}^{*}, \mathrm{G}^{*}\right)}{\partial(\mathrm{H}, \theta)}
$$

where $F(H 1, H 2)=F^{*}(H, \theta)$ and $G\left(H_{1}, H_{2}\right)=G^{*}(H, \theta)$ are continuously differentiable functions. We obtain that $L^{*}(H, q)$ and $\phi^{*}(H, \theta)$ satisfy 


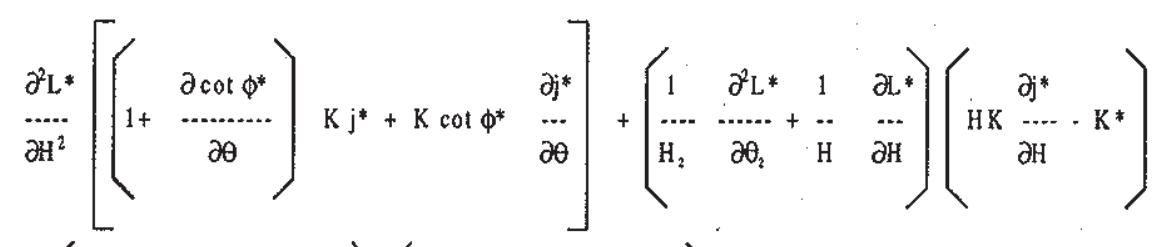

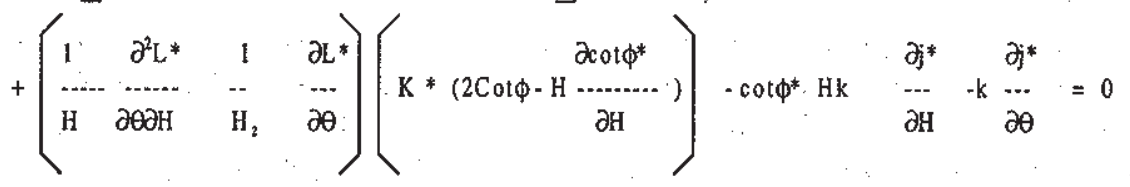

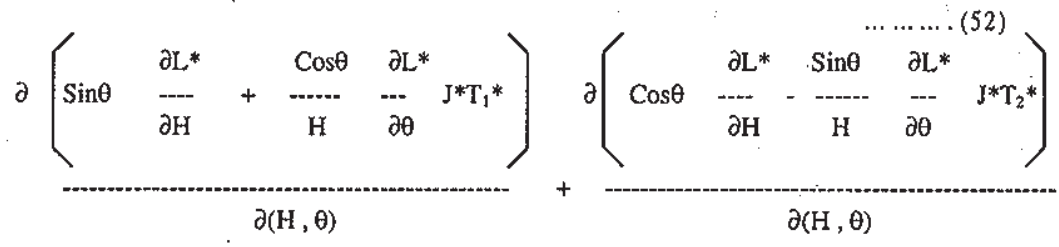

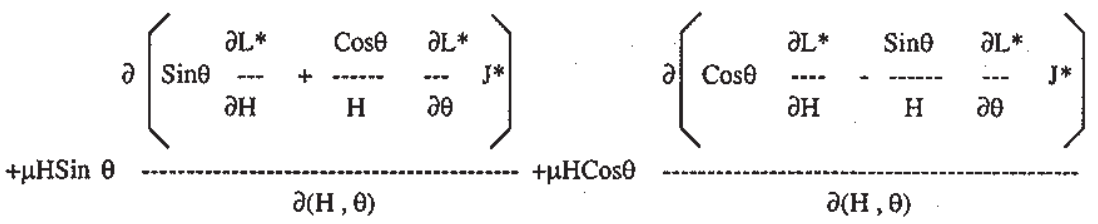
$-\rho k^{*} T_{1} *\left(\operatorname{Sin} \theta \operatorname{Cot} \phi^{*}-\operatorname{Cos} \theta\right)+T_{2}^{*}\left(\operatorname{Sin} \theta+\operatorname{Cos} \theta \cot \phi^{*}\right)=0$

Where

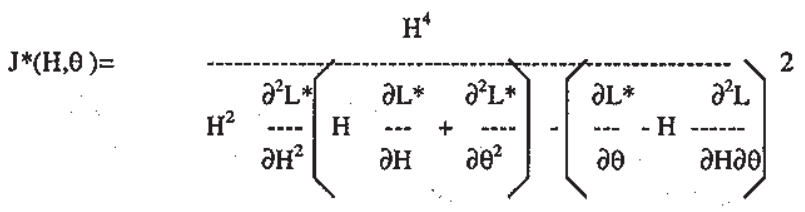

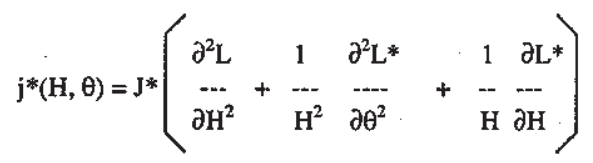

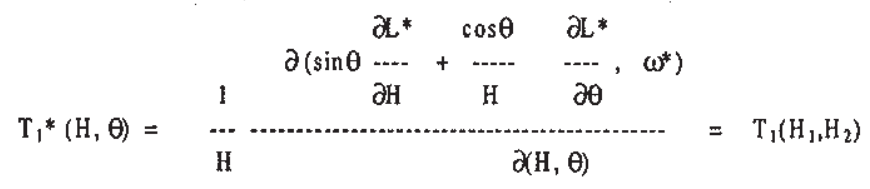




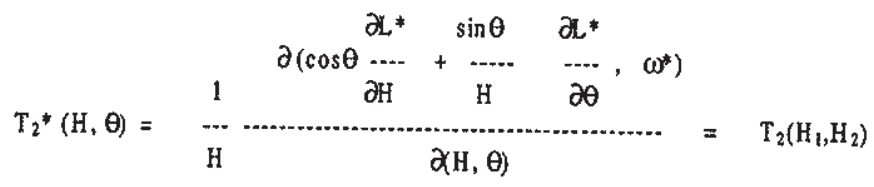

and

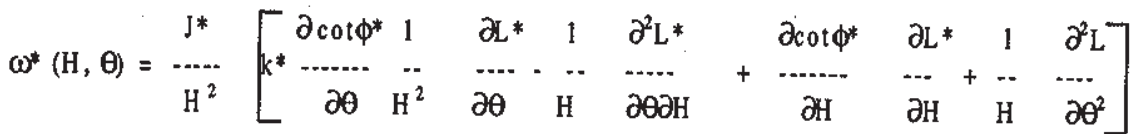

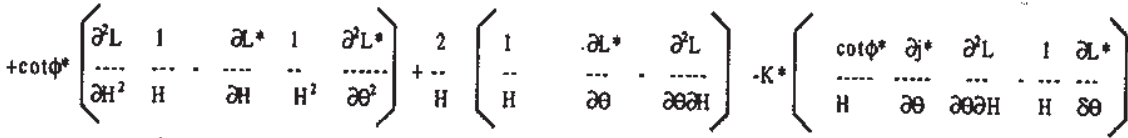

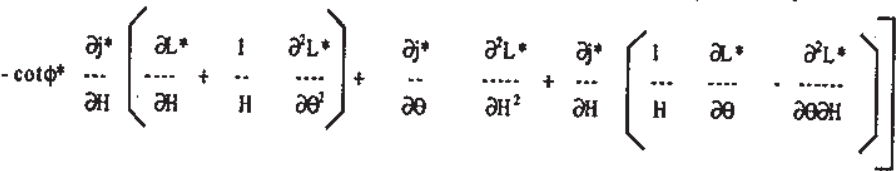

Once a solution $L^{*}=L^{*}(H, \theta), \phi^{*}=\phi^{*}(H, \phi)$ of the system of equations (51) and (52) is determined, we employ

$x=\sin \theta \frac{\partial L^{*}}{\partial \mathrm{H}}+\frac{\cos \theta}{-\ldots} \frac{\partial \mathrm{L}^{*}}{\mathrm{H}} \frac{-}{\partial \theta}$

$y=$\begin{tabular}{ll}
$\sin \theta$ & $\partial \mathrm{L}^{*}$ \\
\hdashline $\mathbf{H}$ & $\frac{--}{\partial \theta}$
\end{tabular}$\quad-\cos \theta \frac{\partial \mathrm{L}^{*}}{\ldots-}$

and $(48)$ to obtain $H_{1}=H_{1}(x, y), H_{2}=H_{2}(x, y)$ in physical plane. The remaining flow variables are then obtained in the physical plane by using the flow equations in the physical plane

\section{Solution}

1. Vertex flow: In this flow, we wish to determine the solution of a flow problem when the Legendre transform function is of the form $L^{*}(H, \theta)=F(H)$ in $(H, \theta)$ co-ordinates. 
$L\left(\mathrm{H}_{1}, \mathrm{H}_{2}\right)=\mathrm{F} \sqrt{\begin{array}{c}2 \\ \mathrm{H}_{1}+\mathrm{H}_{2}\end{array}}$ in $\left(\mathrm{H}_{1}, \mathrm{H}_{2}\right)$ co-ordinates in the hodograph plane.

Let us assume $L^{*}(H, \theta)=F(H)$

to be the Legendre tranform function for the system of equations (52) and (53) such that $F^{\prime}(H)^{1} 0, F^{\prime \prime}(H)^{3} 0$. From equation $(60)$ and $(51)$ we find that $\phi^{*}(H, \theta)$ satisfies

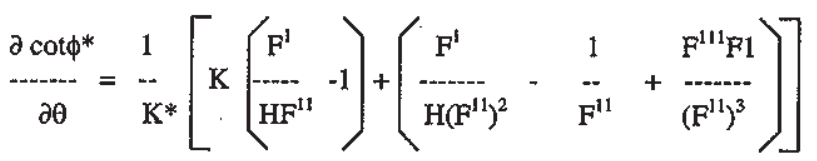

where $\mathrm{j}^{*}=\frac{\mathrm{H}}{\overline{\mathrm{F}^{i}}}+\frac{1}{\mathrm{~F}^{11}}$

As calculated from (55) integration of (61) yields cotф* $=G_{1}(H) \theta+G_{2}(H)$

Where

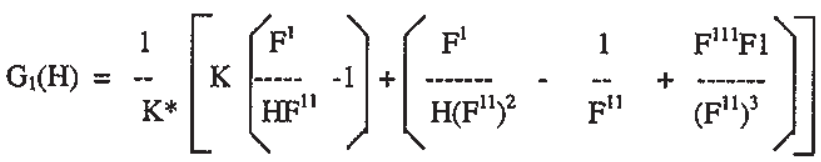

and $\mathrm{G} 2(\mathrm{H})$ is an arbitary constant of $H$. By using $(60)$ and $(63)$ in $(54)$ to $(58)$, we find $\omega^{*}=A(H) \theta+B(H)$

$T_{1}^{*}=\frac{F^{11}}{H} A(H) \sin \theta-\left(\frac{F^{1}}{H} \cos \theta\right)\left(A^{1}(H) \theta+B^{1}(H)\right)$

$\mathrm{T}_{2}{ }^{*}=\underset{\mathrm{H}}{\mathrm{F}^{11}} \mathrm{~A}(\mathrm{H}) \cos \theta-\left(\underset{\mathrm{H}}{\mathrm{F}^{\mathrm{k}}} \sin \theta\right)\left(\mathrm{A}^{\mathrm{i}}(\mathrm{H}) \theta+\mathrm{B}^{1}(\mathrm{H})\right)$

$\mathrm{J}^{*}=\frac{\mathrm{H}}{-\mathrm{F}^{11} \mathrm{~F}^{3}}$

Where 


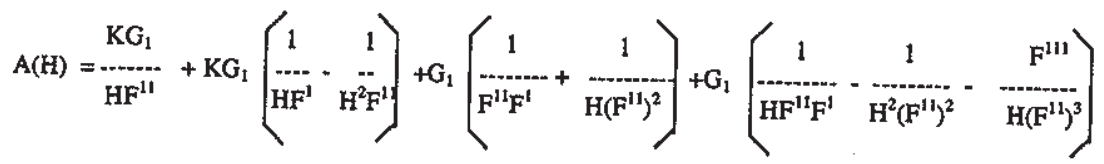

$$
\begin{aligned}
& \mathrm{B}(\mathrm{H})=\frac{\mathrm{KG}_{2}}{\mathrm{HF}^{11}}+\mathrm{KG}\left(\begin{array}{cc}
1 & 1 \\
\hdashline \mathrm{HF}^{1} & \mathrm{H}^{2} \mathrm{~F}^{11}
\end{array}\right)+\mathrm{G}_{2}\left(\begin{array}{cc}
1 & 1 \\
\hdashline \mathrm{F}^{11} \mathrm{~F}^{1} & +\frac{1}{\mathrm{H}^{2}\left(\mathrm{~F}^{11}\right)^{2}}
\end{array}\right)+\mathrm{G}_{2}\left(\begin{array}{ccc}
1 & 1 & \mathrm{~F}^{111} \\
\hdashline \mathrm{HF}^{11} \mathrm{~F}^{1} & \mathrm{H}^{2}\left(\mathrm{~F}^{11}\right)^{2} & \mathrm{H}\left(\mathrm{F}^{11}\right)^{3}
\end{array}\right)
\end{aligned}
$$

Thus $\mathrm{L}^{*}(\mathrm{H}, \theta)$ and cot $\phi^{*}$ given by $(60)$ and $(63)$ respectively, satisfy equation (52) but in order to be a solution of equations (52) and (53) the unknown functions $F(H)$ and $G 2(H)$ must satisfy

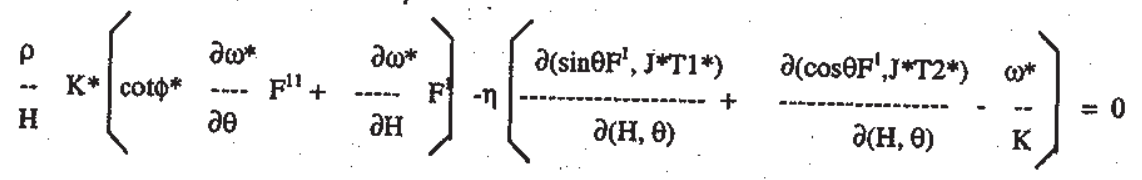

with $i^{*}, \cot \phi^{*}, \omega^{*}, T_{1}{ }^{*}, T_{2}{ }^{*}$ and $J^{*}$ as in $(62)$ to $(68)$ we find that $F(H)=M_{1} H^{2}+M_{2}$, $G_{2}(H)=M_{3}$ is a solution set of $(71)$ such that $F^{\prime}(H) \neq 0, F^{\prime \prime}(H) \neq 0$ where $M_{1} \neq 0$,
$M_{2}$ and $M_{3}$ are arbitrary constants Hence $L^{*}(H, \theta)=M_{1} H^{2}+M_{2}$

$\phi^{*}(H, \theta)=\cot ^{3} M_{3}$

is a solution set of the system of partial differential equations (52) and (53) using the Legendre transform function from (73) and (74) in equation (59) and (65) to (68) expressions for the magnetic component, the vorticity and the current density are obtained as

$$
H_{1}=\frac{-y}{2 M_{1}}, H_{2}=\frac{x}{2 M_{1}}, \quad \omega=0, \quad j=\frac{1}{M_{1}}
$$

The velocity components by using $(73),(74)$ and $(75)$ in (20) are given by

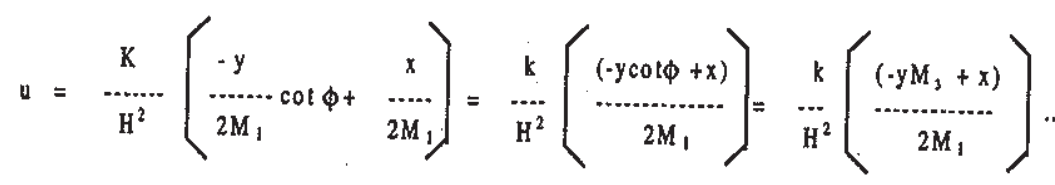


$v=\frac{K}{H^{2}}\left(\frac{\left(\mathrm{xM}_{3}+\mathrm{y}\right)}{2 \mathrm{M}_{1}}\right)$

Finally employing (75) and (76) in (22) and (23), and integrating we find the function $h(x, y)$ and substituting $h(x, y)$ in $(10)$ we find the pressure function as

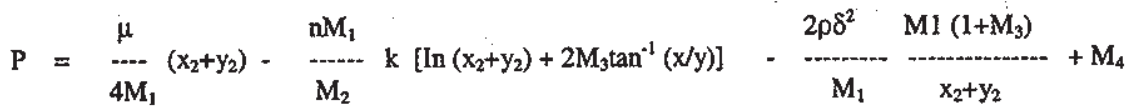

where M4 is an arbitray constant summing up

A variably inclined steady MHD flow through porous media using Hodograph transformation problem with the families of stream lines and magnetic lines given by $x^{2}+y^{2}=$ constant and $M_{3}\left(\ln \left(x^{2}+y^{2}\right)\right)-\tan ^{-1}(x / y)=$ constant

\section{Flow With Magnetic Magnitude Constant on Each Individual Stream Line}

Using (24), equation (27) yields

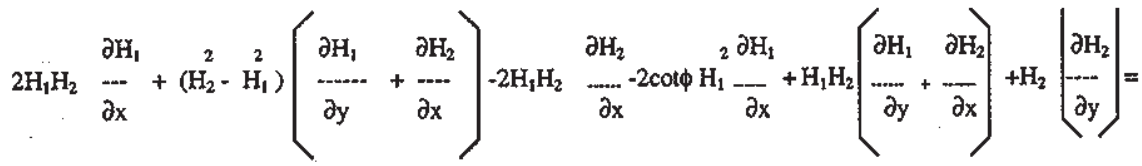

If the magnetic magnitude is constant on each individual stream line then we have

$\mathrm{H} . \nabla \mathrm{H} 2=0$

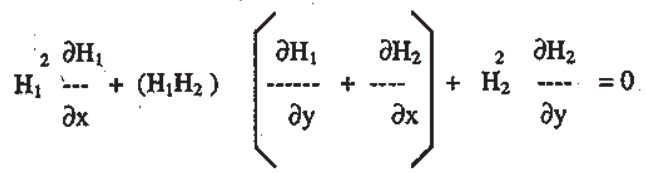

Using (79) in (78), the continuity condition becomes

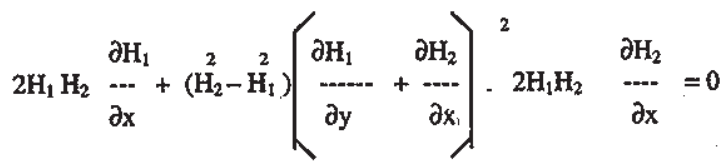


So, if the magnetic magnitude is constant along each individual stream line of an incompressible fluid, then $\mathrm{H}_{1}, \mathrm{H}_{2}$ must satisfy the equation (9), (79) and (80), we discuss thes following coses:

Case 5.1: Let $\mathrm{J} \neq 0$ : In the flow region under consideration let the flow variables $H_{1}(x, y), H_{2}(x, y)$ be such that the transformation Jacobian

$$
\mathrm{J}=\frac{\partial\left(\mathrm{H}_{1}, \mathrm{H}_{2}\right)}{\partial(\mathrm{x}, \mathrm{y})} \neq 0 \text {. I.e. } 0<|\mathrm{J}|<\infty
$$

then from (49) and (50) we get

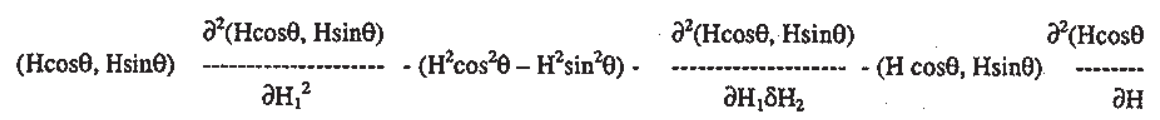

$$
\begin{aligned}
& \text { H } \frac{\partial^{2} L^{*}}{\partial \mathrm{H}^{*} \theta}-\frac{\partial \mathrm{L}^{*}}{\partial \theta}=0 \\
& H^{2} \underset{\partial H^{2}}{\stackrel{\partial^{2} L^{*}}{-H^{2}}} \quad \text {-H } \frac{\partial L^{*}}{\partial \mathrm{H}}-\frac{\partial^{2} L^{*}}{\partial \theta^{2}}=0
\end{aligned}
$$

General solution of (81) is given by,

$L^{*}(H, \theta)=H(\alpha(\theta))+\beta(H)$

where $\alpha(\theta)$ and $\beta(H)$ are arbitrary functions of their arguments. Using (83) in (82) we get

$$
\left(H \beta^{11}-\beta^{1}\right)-\left(\alpha^{11}+\alpha\right)=0 \text { and } \quad H\left(\beta^{11}-\beta^{1}\right)=\left(\alpha^{11}+\alpha\right)=m_{1} \text { (say) }
$$

where $m_{1}$ is a constant and primes denote the differentiation with respect to the arguments

Now $H \beta^{11}-\beta^{1}=m^{1}$ gives $\beta(H)=m / 2 H^{2}-m_{1} H+m_{2}$

where $m_{1}$ and $m_{2}$ are constants and

$\alpha^{11}+\alpha=m_{1}$ gives $\alpha(\theta)=A \cos \theta+B \sin \theta+m_{1}$

where $A$ and $B$ are arbitrary constant. Substituting (84) and (85) in (83) gives 
$L^{*}(H, \theta)=H\left(A \cos \theta+B \sin \theta+m_{1}\right)+1 / 2 m^{2}-m_{1} H+m_{2}$

The above with the help of (49) and (50) can be written as

$\mathrm{L}\left(\mathrm{H}_{1}, \mathrm{H}_{2}\right)=\mathrm{AH}_{1}+\mathrm{BH}_{2}+(1 / 2 \mathrm{~m})\left(\stackrel{2}{2} \mathrm{H}_{1}+\mathrm{H}_{2}\right)+\mathrm{m}_{2}$

Then $x=B+\mathrm{mH}_{2}, y=-\left(A+\mathrm{mH}_{1}\right), \mathrm{H}_{2}=(1 / m)(x-B), H_{1}=-(y+A) / m$

$$
j=\frac{\partial((1 / m)(x-B))}{\partial x}-\frac{\partial(-(y+A) / m)}{\partial y}
$$

$j=2 / m$

$$
\begin{aligned}
& u=k m \quad \frac{(x-B)-(y+A) \cot \phi}{(x-B)^{2}+(y+A)^{2}} \text { and } \\
& v=k m \quad \frac{(x-B) \cot \phi+(y+A)}{(x-B)^{2}+(y+A)^{2}} \\
& h=h_{0}+\frac{\rho k}{m^{2}}\left[(x-B)^{2}+(y+A)^{2}\right]+\frac{\eta}{k m}(A x-B y+x y)
\end{aligned}
$$

If $K \rightarrow \infty$, i.e. porous media is removed, we get all the results of Sattar and Chand na for velocity vector. The pressure is given by

$P=p_{0}+\frac{\rho k}{m^{2}}\left(1 / 2\left(x^{2}+y^{2}\right)\right)+(A y-B x)+\frac{\eta}{k m}(A x-B y+x y)$

and stream lines are given by $(x-B)^{2}+(y+A)^{2}=$ constant which represent concentric circles whose centre at $(B,-A)$.

Case 5.2:. Let $J=0$ : Let the flow variables $H I(x, y), H 2(x, y)$ be such that in the region of flow the Jacobian

$$
J=\frac{\partial\left(\mathrm{H}_{1}, \mathrm{H}_{2}\right)}{\partial(\mathrm{x}, \mathrm{y})}=0
$$


In such a case either $\mathrm{H}^{1}$ is a function of $\mathrm{H}_{2}$ or $\mathrm{H}_{2}$ is a function of $\mathrm{H}_{1}$. We consider $\mathrm{H}_{2}$ as a function of $\mathrm{H}_{1}$.

$\mathrm{H}_{2}=\phi\left(\mathrm{H}_{1}\right)$

where $\phi$ is an arbitary constant of $\mathrm{H}_{1}$. Using (92) in equation (30), we obtain

$$
\frac{\partial \mathrm{H}_{1}}{--\mathrm{x}^{1}}+\phi^{1}\left(\mathrm{H}_{1}\right) \frac{\partial \mathrm{H}_{1}}{\partial \mathrm{y}}=0
$$

Eliminating $\left(\partial \mathrm{H}_{1} / \partial \mathrm{x}\right)$ from (93) and (80) we get

$\left(\mathrm{H}_{1}+\phi \phi^{1}\right)\left(\mathrm{H}_{1} \phi^{1}-\phi\right) \mathrm{H}_{1}\left(\partial \mathrm{H}_{1} / \partial \mathrm{y}\right)=0$

From the above we have the following:

case 5.2.1: $\frac{\partial \mathrm{H}_{1}}{\partial \mathrm{y}}=0, \quad \mathrm{H}_{1} \phi^{1}-\phi \neq 0$

case 5.2.2: $\mathrm{H}_{1} \phi^{1}-\phi=0$

case 5.2.3: $\mathrm{H}_{1}+\phi \phi^{1}=0$

case 5.2.4: $\frac{\partial \mathrm{H}_{1}}{\partial \mathrm{y}}=0, \quad$ implies $\frac{\partial \mathrm{H}_{1}}{\partial \mathrm{x}}=0$

i.e. $H_{1}=k_{1}$ and $H_{2}=k_{2}$ hence the solutions are $\omega=0, j=0, H_{1}=$ constant, $\mathrm{H}_{2}=$ constant,

$h=h_{0}-(\eta / k m)\left(k_{1} x+k_{2} y\right)$

$P=P_{0}-(\eta / k m)\left(k_{1} x+k_{2} y\right)$

$P_{0}=$ constant $=h_{0}=(\rho / 2)\left(k_{1}+k_{2}\right)$

The equation of stream line is given by

$K_{2} x \cdot K_{1} y=$ constant and represents a parallel straight line. 
Case 5.2.2: $\quad H_{1} \phi^{1}-\phi=0$ implies $\phi=k_{3} H_{1}$ i.e. $\phi_{1}=k 3$.

i.e. $\frac{\partial \mathrm{H}_{1}}{\partial \mathrm{x}}+\mathrm{k}_{3} \frac{\partial \mathrm{H}_{\mathrm{I}}}{\partial---}=0$

General solution of the equation is given by $H_{1}=f\left(k_{3} x-y\right)$ and $H_{2}=k_{3} f\left(k_{3} x-y\right)$ . which implies $f 1\left(k_{3} x-y\right)=0$

Case 5.2.3: Put restriction on $\phi$ such that $H_{1}+\phi^{2}=$ constant

hence $H_{1}=f\left(k_{3} x-y\right)$ and $H_{2}=k_{3} f\left(k_{3} x-y\right), \omega=0, j=0$

$\mathrm{u}=\frac{\mathrm{k}\left(\cot \phi-\mathrm{k}_{3}\right)}{\left(1+\mathrm{k}_{3}\right) \mathrm{f}}, \mathrm{v}=\frac{\mathrm{k}\left(1+\cot \phi \mathrm{k}_{3}\right)}{\left(1+\mathrm{k}_{3}\right) \mathrm{f}}, \mathrm{h}=\mathrm{h}_{0}-(\eta / \mathrm{k})\left(\mathrm{xf}+\mathrm{k}_{3} \mathrm{yf}\right)$

22

$\mathrm{p}=\mathrm{p}_{0}-(\rho / 2)\left(1+\mathrm{k}_{3}\right) \mathrm{f}-(\eta / \mathrm{k}) \mathrm{f}\left(\mathrm{x}+\mathrm{k}_{3} \mathrm{y}\right)$

and stream line is given by $k_{3} x-y=$ constant and are parallel straight lines.

\section{Conclusion}

If is shown that when the velocity and magnetic vectors of MHD flow are constantly variably inclined then the streamlines are concentric circles and the magnetic lines are spirals. These are shown by means of graphs (figures $3 \& 4$ ). Also graphs are plotfed for velocity components $\mathrm{U}, \mathrm{v}$ against $\mathrm{Hl}$ ( figures $1 \mathrm{a}, \mathrm{lb} \& 2$ ). In figure $1 \mathrm{a}$, the curves almost coincide at angles $45^{\circ} \& 75^{\circ}$. In figure $1 \mathrm{~b}$, the curves almost coincides at angles $70^{\circ} \& 67^{\circ}, 68^{\circ} \& 65^{\circ}$ respectively. But however for angle $66^{\circ}$ the curve steeps above. This is because velocity and magnetic vectors are variably inclined.

\section{References}

1. Chandna. O. P. Toews.H. and Nath.VI.(1975) Can. J. Phys. 53.

2. Ram.G. and Mishra.R.S. (1977) Ind. Jour.Pure and applied maths 8,161

3. Sattar M.A. and Chandna O.P. (1988) Jour.maths.phy.sc. 22,3

4. Yamamoto K. (1971) Jour.phy.soc. Japan 31, 572 
5. L.D.Landav and E.M.Lifshitz, Fluid mechanics, Pergamon press, Oxford. Vol.6 (1959).

6. C.S.Bagewadi and Siddabasappa (1991) Ganitha, Lucknow, Vol.42, 13-21.

7. C.S.Bagewadi and Siddabasappa (1993) Bulletin of Cal. Math. Soc. vol 85, 513-520.

8. K.K.Singh and D.P.Singh (1993) Bull. Cal. Math. Soc 85, 255-262

9. C.S.Bagewadi and Siddabasappa (1991), Journal of karnataka University, Sci. Dhanwad, Vol.35, 131-146.

10. O.P.Chandina and Nguyen P.V. (1992) Int. J .Engg. Sci. 30,69.

11. Siddabasappa \& C.S.Bagewadi (1992) Kar.Uni.Science Journal, Dharwad, Vol.36, 103. 113.

12. C.S.Bagewadi \& Siddabasappa (1995) Bulletin of Calcutta, Mathematical Society, Calcutta, Vol .87, 41$\}-485$.

13. Yamamoto. K. (1973) Jour.Phys. Soc. Japan 34, 814:

14. O.P.Chandna, R.M. Barron and K.T.Chew (1982), J.Engg. Math., Vol.16, 223-243.

15. R.M.Barron and O.P.Chandna (1981), J.Engg. Math., Vol.5, No.3, 211-220. 


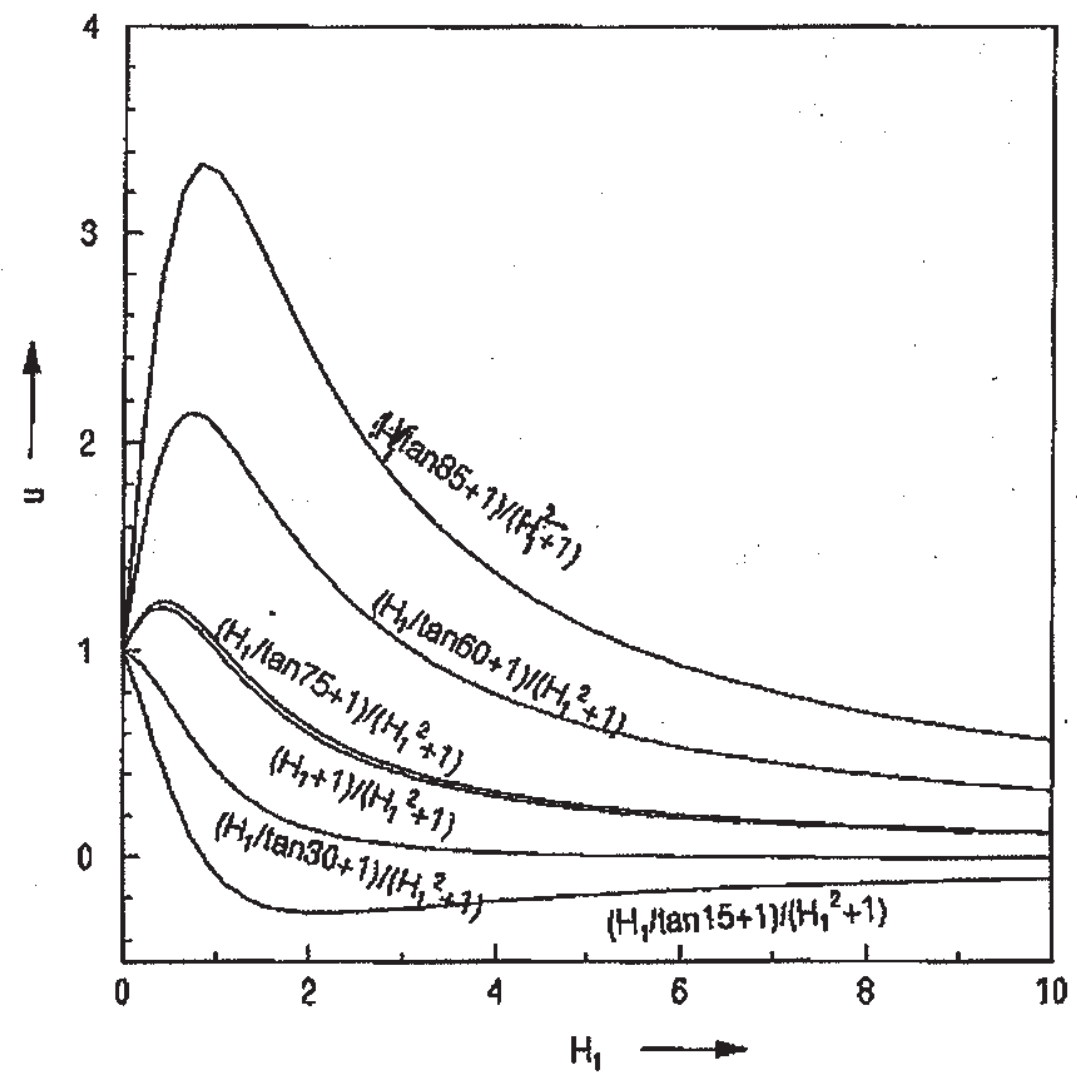

Fig. Ia Variation of $\mathrm{u}$ with respect to $\mathrm{H}_{1}$

$$
\begin{gathered}
k=1, H_{2}=1 \\
u=\frac{k}{H^{2}}\left(H_{1} \cot \phi+H_{2}\right)
\end{gathered}
$$




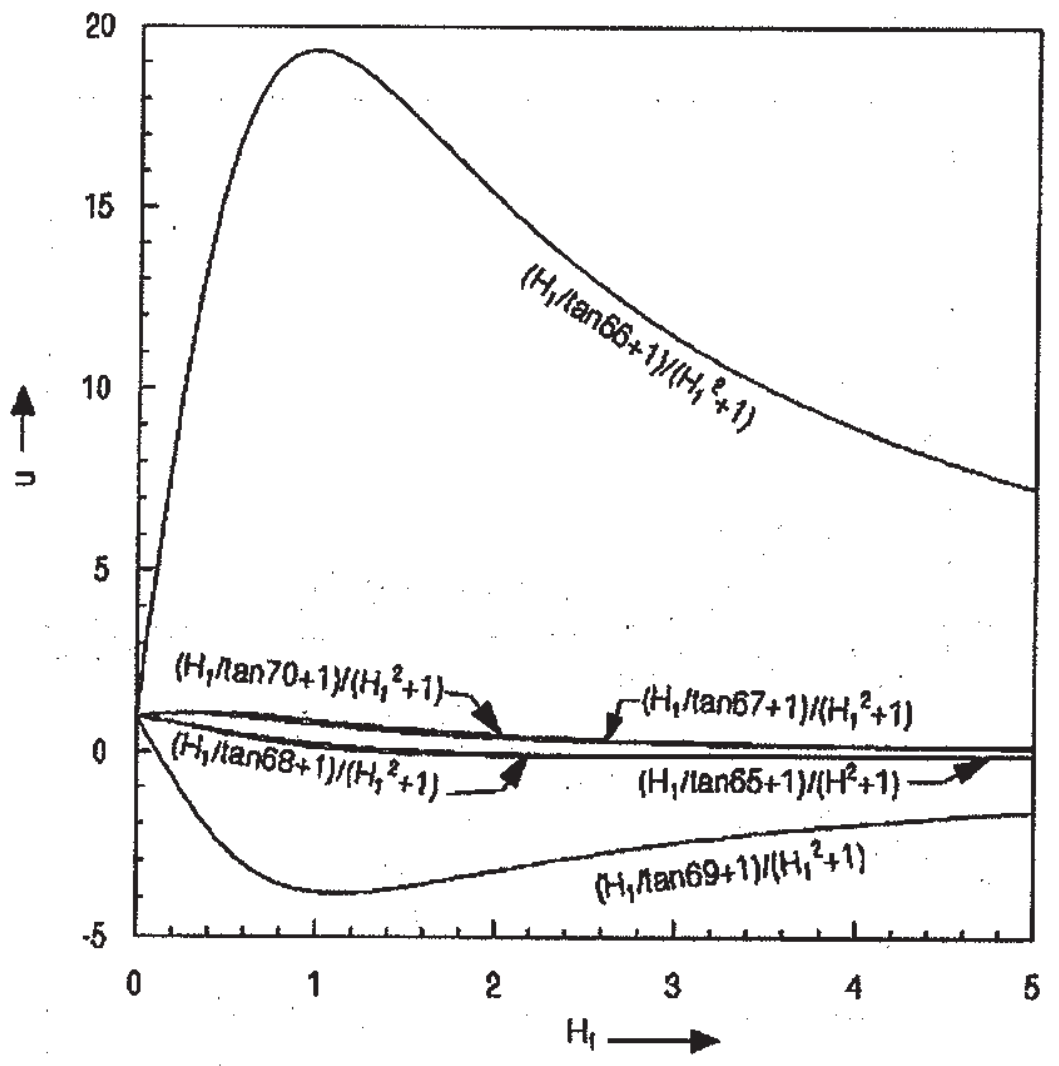

Fig. $1 \mathrm{~b}$ Variation of $\mathrm{u}$ with respect to $\mathrm{H}_{1}$

$$
\begin{gathered}
k=1, H_{2}=1 \\
u=\frac{k}{H^{2}}\left(H_{1} \cot \phi+H_{2}\right)
\end{gathered}
$$




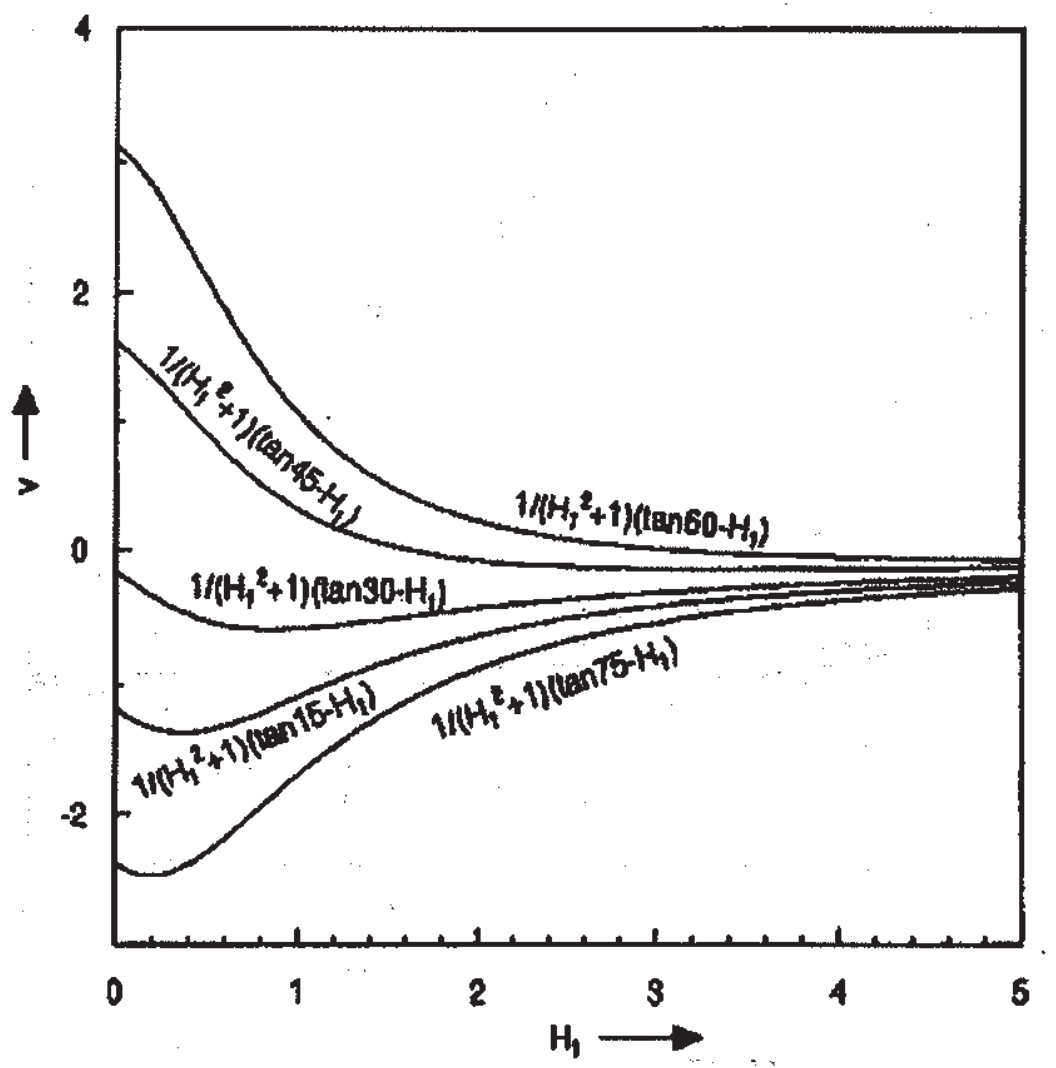

Fig. 2 Variation of $v$ with respect to $H_{1}$

$$
\begin{gathered}
k=1, H_{2}=2 \\
V=\frac{k}{H^{2}}\left(H_{2} \cot \phi-H_{1}\right)
\end{gathered}
$$




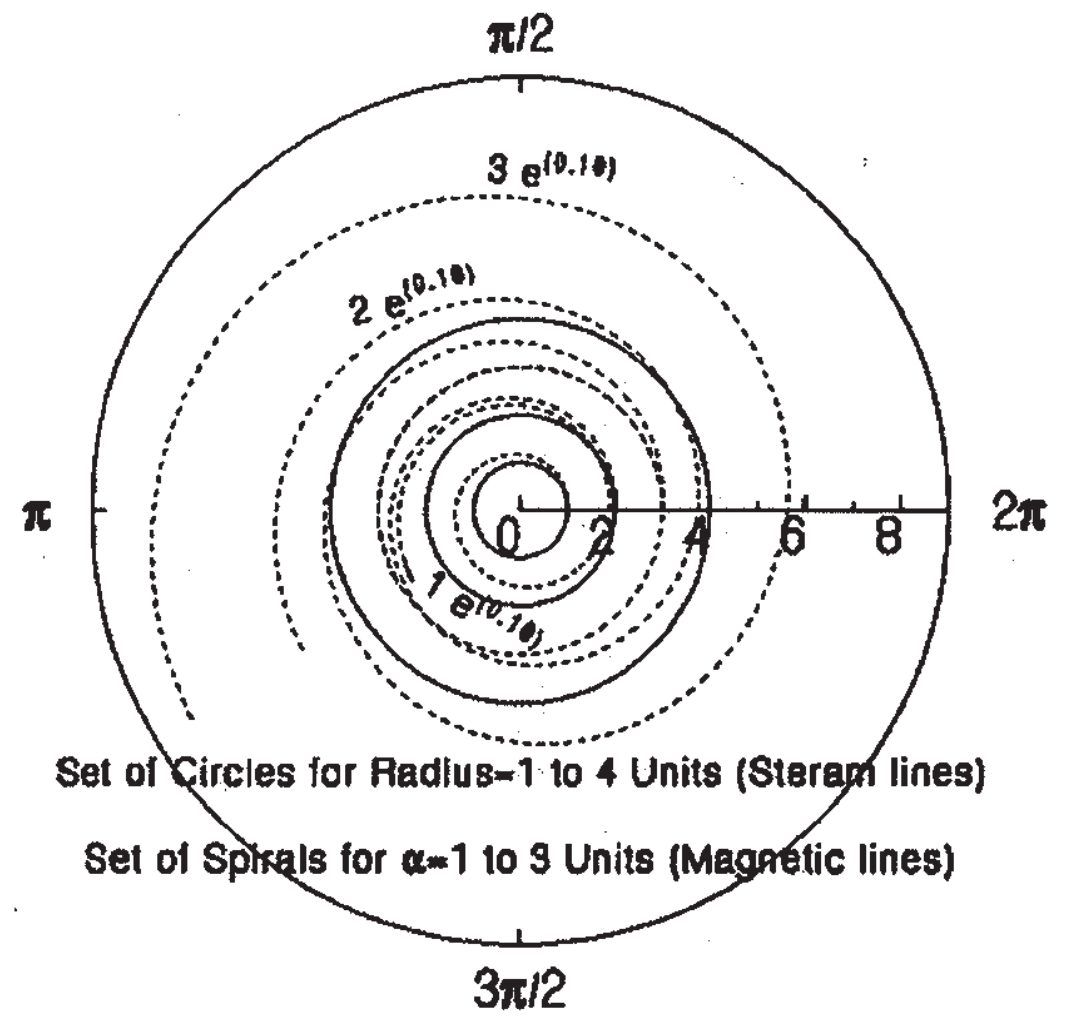

Fig. 3 Variation of Stream lines and Magnetic lines 


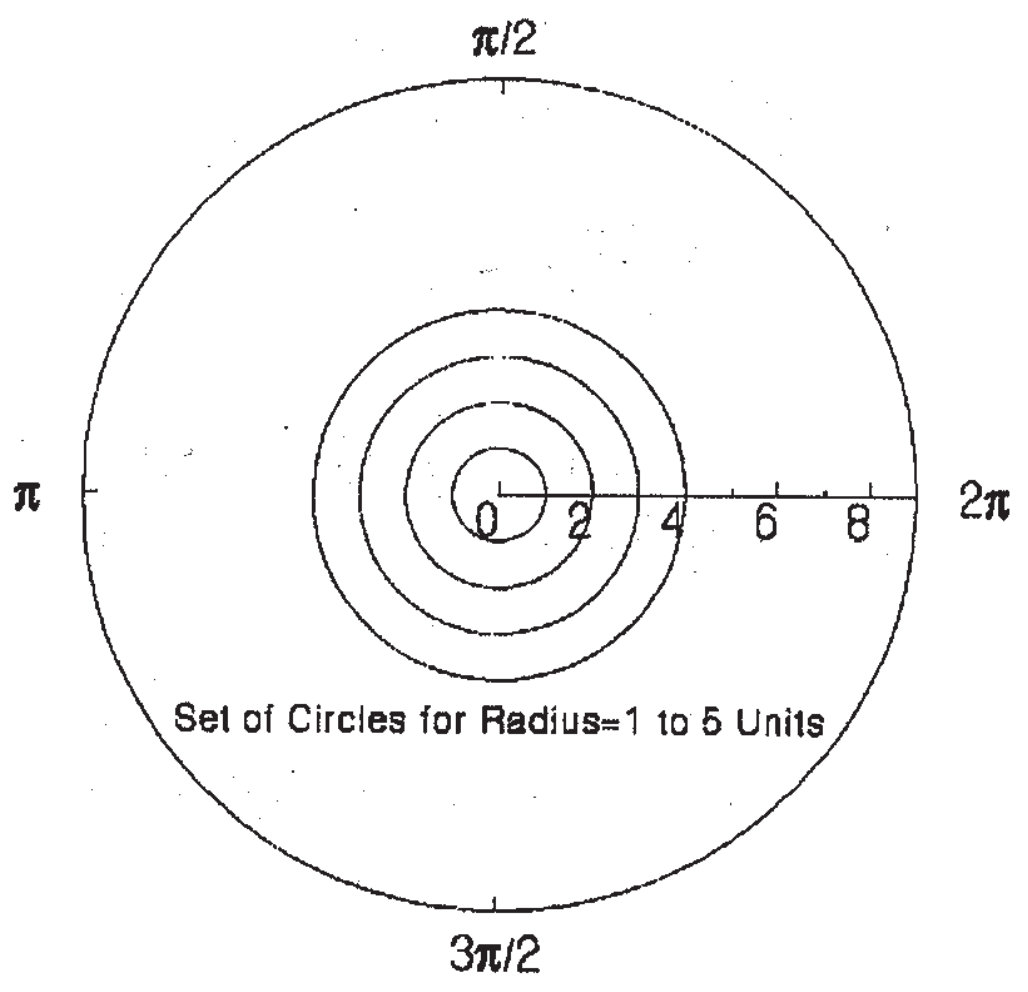

Fig. 4 Variation of $u^{2}+v^{2}=\sqrt{\left(x^{2}+y^{2}\right)}$ 